\title{
Profile-based Service Continuity Framework for N-Screen Service
}

\author{
Young-Sik Chung, Eui-Hyun Paik \\ Social computing research team \\ ETRI, Daejeon, Korea \\ Woo-Seop Rhee \\ Department of Multimedia Engineering \\ Hanbat National University, Daejeon, Korea
}

\begin{abstract}
The dynamic adaptation between various service environments using the application profiles for the service continuity is a key issue of the profile-based service continuity framework (PSCF) for N-screen service using next generation networks. PSCF offers an optimized service framework for providing continuous user services, which are multimedia video streaming, educational broadcasting, game, etc., using the various devices that are not restricted by the service environment of the user. This paper specifies the functional model of PSCF, service scenario and explains the experimental results of the service continuity for $N$-screen service using PSCF.
\end{abstract}

Keyword: Profile, Profile-based service continuity framework, $N$-screen

\section{INTRODUCTION}

As the application service becomes more and more diversified in accordance with the varying service environment, the adaptation service between different service environments will need to overcome the restricted service realization. For example, in ubiquitous computing environment, many types of mobile devices are used, and they have different system specifications from those of others. In that case users move from one service environment to another, they want to be provided the continuous service regardless of the devices they use. This kind of continuous service is one example of the $\mathrm{N}$-screen service.

To support this service continuity, application service profiles are registered in the service provider according to each application service. They have different attributes. A user wishing to change his service environment should be provided with continuous service to adapt the service profiles having different attributes. It is required that advanced application services of next generation networks (NGN) overcome some of the service restriction of the different service environment. Therefore, Profile-based Service Continuity Framework (PSCF) provides continuous service using the application service profiles, which is adapted to the changed service environment such as user devices.

Figure 1 presents the conceptual model of PSCF for N-screen

* Corresponding author. E-mail : wshree@hanbat.ac.kr Manuscript received Dec. 05, 2011 ; accepted Jan.31, 2012 service. PSCF provides continuous user service when a user moves from PC to PDA and/or Mobile Phone. The pre-encoded data formats of contents according to the different devices for the user service are provided by the Contents / Broadcast / Internet service provider.

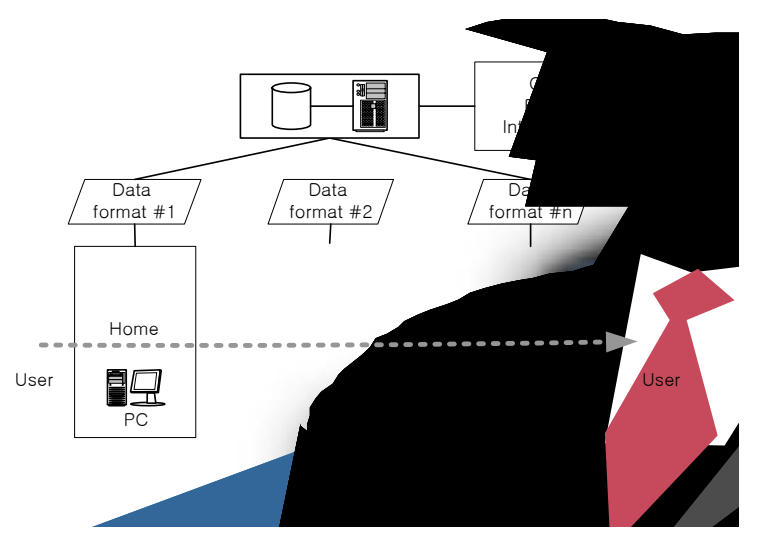

Fig. 1. Conceptual model of PSCF for N-screen service

The rest of this paper is organized as follows. Section 2 describes the related research works, section 3 and section 4 describe the functional model and functional capabilities of PSCF using NGN. Section 5 explains the functional procedures of PSCF function and section 6 explains about experimental results. Finally, section 7 makes conclusion. 


\section{RELATED WORK}

There are many exiting researches related to service mobility [1], [3], [4]. In [2], it presents the method of solution that provides 3 screens on multiple internet video streaming. This approach enables heterogeneous terminals to access manageable P2P framework and presents topology configuration for improved UDP transport and intelligent contents distribution. However there is no concept of contents synchronization for the service continuity during service mobility. There are also discussed in various perspectives on the focus of the exiting terminal or host mobility [5]-[7]. In [6], for providing the continuous internet connectivity, mobile IP protocol was proposed with the host's location registration.

In the view point of User mobility [8]-[12], these projects indicate the problem about using middleware for mobile IP and mobile network. In addition, there are several approaches that the mobility of streaming service. According to approaches dealing with different bandwidth-based on-demand service, it is the issue that how to manage the heterogeneous terminal. In [13], it presented continuous computing concept and application session handoff architecture. This enabled contents adaptation to the heterogeneous terminal for continuous computing. However, it did not provide service continuity for multiple users. In [14], the overlay concept and the contents delivery method in the P2P streaming are considered as design elements. Gossip protocol for overlay configuration and swarm-based protocol for contents delivery were used for the implementation.

Many works are focused on the contents delivery and contents adaptation at the various layers. In this paper, we propose a service synchronization framework for service continuity and synchronization on the heterogeneous terminals in aspect of user mobility.

\section{FUNCTIONAL MODEL OF PSCF USING NGN}

Based on the NGN framework architecture of ITU-T SG13 [15], the functional model of PSCF uses the functions and functional entities of RACF (Resource and Admission Control Function) [16], NACF (Network attachment control functions) [17], MMCF (Mobility management and control framework) [18], and SCF (Service Control Function). The PSCF functions are added in the application part of the NGN framework architecture, and they communicate with NGN via the application network Interface (ANI) as shown in (a) or via the service network interface (SNI) shown in (b) of Figure 2. ANI interfaced PSCF functions are provided by the ISP (Internet Service Provider) and SNI interfaced PSCF functions are provided by the $3^{\text {rd }}$ party service provider.

This figure also presents an overview of the functional model. The PSCF consists of the Application adaptation service management function, Profile control function and application service profiles, Adaptation service control function, Service environment adaptation function and Application adaptation service interface function using NGN as shown in Figure 2.

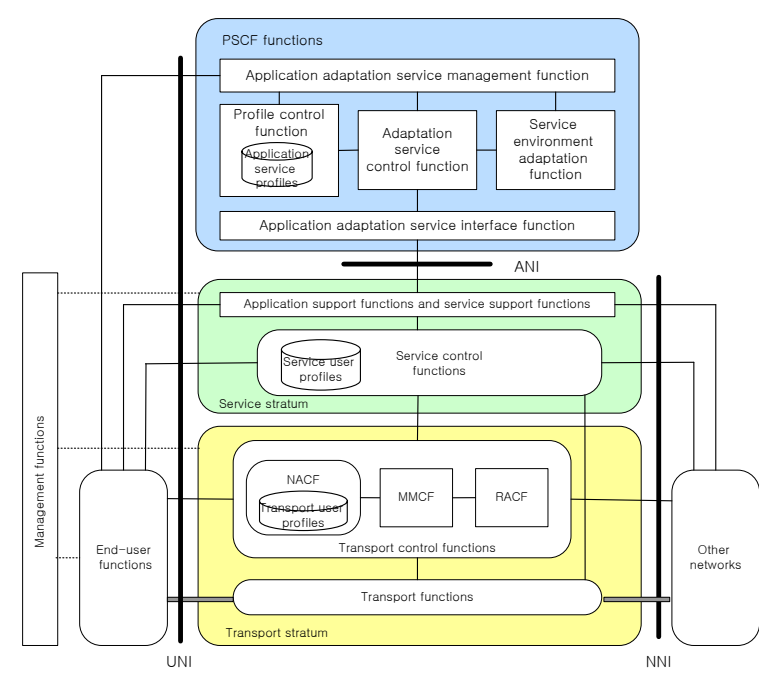

(a) Functional model with ANI interface

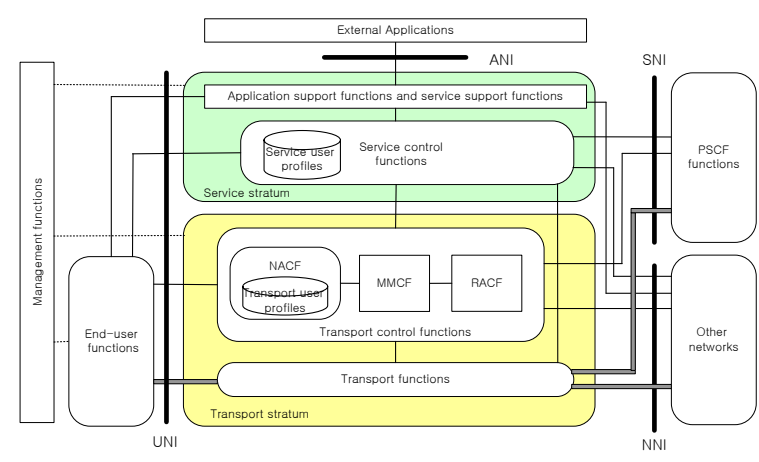

(b) Functional model with SNI interface

Fig. 2. Functional model for PSCF using NGN

\subsection{Application adaptation service management function}

The application adaptation service management function is used to process user authentication and authorization. Using user information, the application adaptation service management function requests the new continuous service to the adaptation service control function. This function also provides the required service information to the user for continuous service and performs the following functions.

- Service registration, service deletion and service information update.

- User registration, user deletion and user information update.

- Service session management.

- Interface with user device.

\subsection{Adaptation service control function}

The adaptation service control function aims at recommending the proper services to the user to adapt to the current user service environments. To do this, it manages the service history using the application service profiles. The adaptation service control function also provides the device information to the service environment adaptation function for the handling of media conversion with profile adaptation. 


\subsection{Service environment adaptation function}

The service environment adaptation function is used to adapt the user service to the new service environment using the appropriate attributes pre-registered in the application service profile. It handles the adaptation of the profile information and provides a proper execution environment according to the control of the adaptation service control function.

\subsection{Profile control function and application service profiles}

The profile control function is used to manage the attributes related to the application service, user, devices and session. It also provides the interface between the application service profiles and other function entities. The application service profiles store the user information, session information, device information and service information used for providing continuous user services. The detail descriptions of each profile are provided in Table 1.

Table 1 Application service profiles

\begin{tabular}{|c|l|}
\hline $\begin{array}{c}\text { User } \\
\text { profile }\end{array}$ & $\begin{array}{l}\text { This profile stores the personal data } \\
\text { including ID, name, address, password, } \\
\text { affiliation, and preferences and/or interests } \\
\text { of users as well as the history of the user's } \\
\text { selection of service. }\end{array}$ \\
\hline $\begin{array}{c}\text { Session } \\
\text { profile }\end{array}$ & $\begin{array}{l}\text { This profile stores the description of } \\
\text { sessions from user log-in and services } \\
\text { provided and log-in status of user, } \\
\text { connection status and period and device } \\
\text { used. }\end{array}$ \\
\hline $\begin{array}{c}\text { Device } \\
\text { profile }\end{array}$ & $\begin{array}{l}\text { This profile stores the description of the } \\
\text { device such as type, vendor, model, and } \\
\text { hardware and software specifications. }\end{array}$ \\
\hline $\begin{array}{c}\text { Service } \\
\text { profile }\end{array}$ & $\begin{array}{l}\text { This profile stores the description of } \\
\text { services including name, provider, language } \\
\text { used, and hierarchy of service categories } \\
\text { and position of each service, search tags of } \\
\text { services and requirements of services for } \\
\text { the proper delivery of service on devices. }\end{array}$ \\
\hline
\end{tabular}

\subsection{Application adaptation service interface function}

The application adaptation service interface function provides interaction between the PSCF functions and application support function of the service stratum via ANI. It also supports the interaction between the PSCF functions and service control functions, transport control functions, and transport functions via SNI. It offers an exchange protocol for a well determined dialogue and the requested format used in the exchange of control message and communication of the user context to the other entities.

\section{FUNCTIONAL CAPABILITIES OF PSCF}

User devices use PC with web browser or PDA, Cellular Phone, and Smart Phone with GUI client for the service interface. User devices also have the service environment adaptation client function for service execution engine downloading from the service environment adaptation function. Figure 3 presents detailed functional model of user device and interface with PSCF functions.

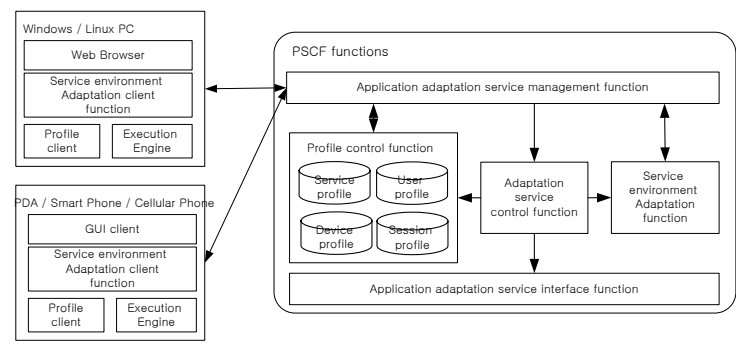

Fig. 3. Detailed functional model of user device and interface with PSCF function

\subsection{Service and User registration / management}

\subsubsection{Service registration, deletion, and update}

The service registration of the PSCF functions is performed by the application adaptation service management function through the service registration page. The service provider also registers the service execution engine for the registered service. The registered service execution engine information provides to the service environment adaptation function for service execution engine downloading. The registered service information is stored in the service profile, which optionally includes:

- Service description

- Service category

- Service keyword

- Service execution engine

The service deletion of the PSCF functions is performed by the application adaptation service management function through the service list page. The application adaptation service management function deletes the requested service in the service profile. It also supports the service update function. This function lists the registered services and service execution engines.

\subsubsection{User registration, deletion and update}

The user registration of the PSCF functions is performed by the application adaptation service management function through the user registration page of the user device. the user inputs the name, user ID, password, area of interest, etc, for the user information. The registered user information is stored in the user profile.

The user deletion of the PSCF functions is performed by the application adaptation service management function through the user deletion page of the user device. The application adaptation service management function deletes the requested user in the user profile. It also supports the user update function. This function lists the registered users and shows user information using the user ID.

\subsection{User preference service recommendation}

User preference service recommendation is performed by the application adaptation service management function, which 
provides the following lists to the user device using the user profile and service profile.

- Recently used service.

- Frequently used services by the user.

- User preference recommended services

For the user preference recommended service, the adaptation service control function provides preference service using the service usage pattern and service category of the user. The user preference recommended services are passed to the application adaptation service management function for listing on the user device.

\subsection{Service execution engine download}

Service execution engine download is performed by the service environment adaptation function for the configuration of a specific service execution environment in the user device.

When requested by a user for a specific service, the application adaptation service management function checks the execution environment of the requested user device using the user profile and service profile. If a user device needs the execution environment of the requested service, the application adaptation service management function informs the adaptation service control function, which then extracts the proper execution engine using the device profile and the session profile and informs the service environment adaptation function of this execution engine information. The service environment adaptation function downloads the service execution engine to the user device.

\subsection{Session management}

\subsubsection{Login session management}

When a user logs in, user authorization is performed by the application adaptation service management function, and information of the login user is added to the session profile. This login session information provides the current user lists to the application adaptation service management function.

\subsubsection{Service execution engine session management}

When a user selects a specific service, and after the service execution engine is downloaded, the adaptation service control function creates a service execution engine session in the session profile, which provides the list of services and service execution engines to the application adaptation service management function.

\section{FUNCTIONAL PROCEDURES OF PSCF}

\subsection{Service registration and release}

Figure 4 shows the functional procedure when a service is registered and released. In this procedure, the application adaptation service management function registers/releases a service and a service execution engine to the service profile.

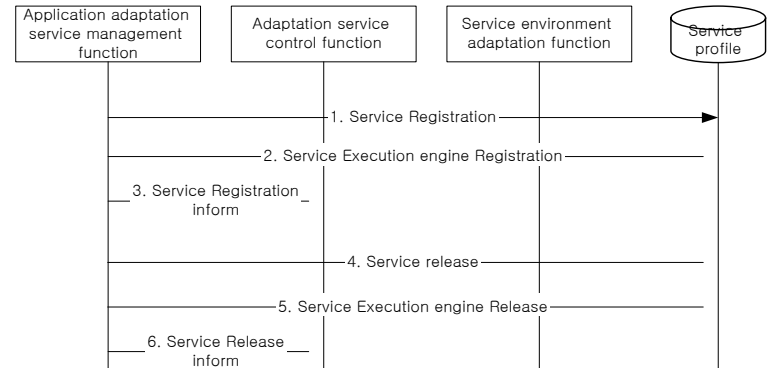

Fig. 4. Functional procedures for service registration and release

Details of procedure are as follows:

1) The application adaptation service management function creates the service information with the service description, service category, service keyword, etc. and requests the addition of service information to the service profile through service registration.

2) The application adaptation service management function also registers the related service execution engine to the service profile through service execution engine registration.

3) After service and service execution engine registration, the application adaptation service management function informs the adaptation service control function of this service profile.

4) The application adaptation service management function deletes the requested service information from the service profile using service release.

5) The application adaptation service management function also releases the related service execution engine from the service profile through service execution engine release.

6) After the service and service execution engine are released, the application adaptation service management function informs the adaptation service control function of this released service information.

\subsection{User registration and release}

Figure 5 illustrates the functional procedure when a user is registered and released. In this procedure, the user registers/releases the user information to the user profile.

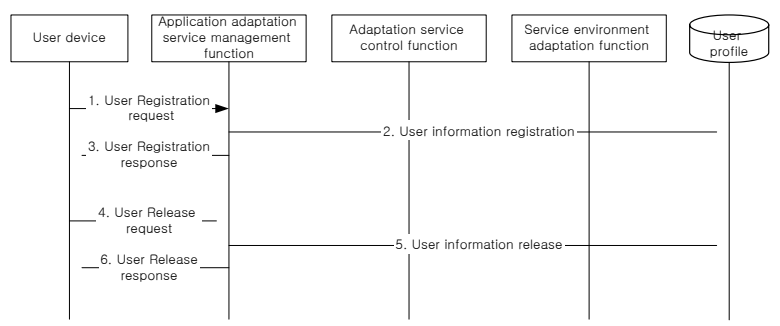

Fig. 5. Functional procedures for user registration and release

Details of procedure are as follows:

1) When a user requests a user registration through the user device, the user registration request message with user information (user ID, name, password, address, etc.) is sent to the application adaptation service management function. 
2) The application adaptation service management function requests the addition of user information to the user profile using the user information registration.

3) The application adaptation service management function sends the user registration response message to the user device.

4) When a user requests release in the user device, the user registration release message with user ID is sent to the application adaptation service management function.

5) The application adaptation service management function requests the deletion of user information to the user profile through user information release.

6) The application adaptation service management function sends the user release response message to the user device.

\subsection{User login}

Figure 6 presents the functional procedure when a user logs in. In this procedure, the application adaptation service management function performs authorization and registers user login session information to the session profile.

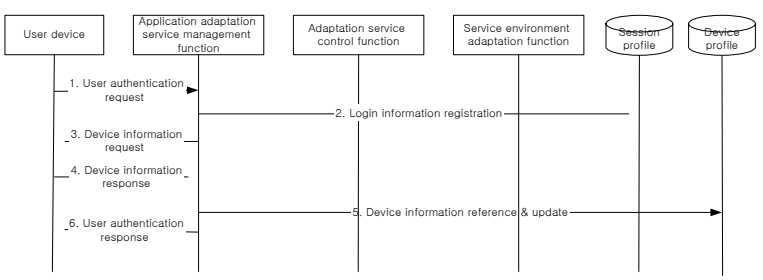

Fig. 6. Functional procedure for user login

Details of procedure are as follows:

1) When a user logs in, the user device sends the user authorization request message to the application adaptation service management function.

2) After the processing of authorization, the application adaptation service management function requests the addition of user login information with user ID, IP address, etc., to the session profile using login information registration.

3) The application adaptation service management function requests the device information of the login user device.

4) The user device sends the device information response message to the application adaptation service management function.

5) Using this device information, the application adaptation service management function references and updates the device profile about the login user.

6) The application adaptation service management function sends the user authorization response message to the user device.

\subsection{Service execution engine download}

Figure 7 shows the functional procedure when a service execution engine is downloaded. In this procedure, a user selects an available service and the necessary service execution engine is downloaded to user device from the device profile for the execution of the selected service.

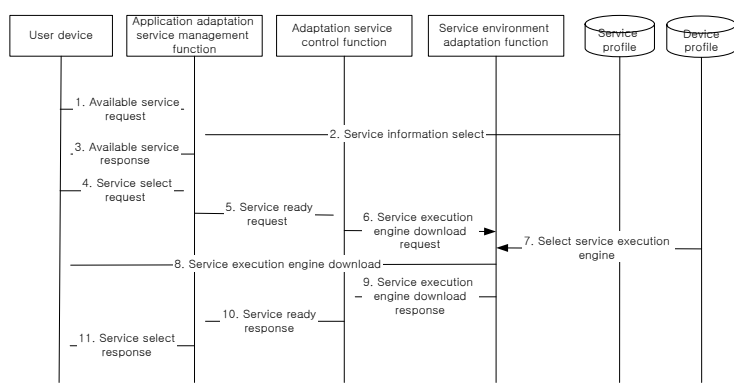

Fig. 7. Functional procedure for service execution engine download

Details of procedure are as follows:

1) A user requests the available services with the available service request message.

2) The application adaptation service management function selects the available services from the service profile

3) The application adaptation service management function provides the selected available services using the available service response message.

4) When selecting a service from the available services, a user sends the service select request message to the application adaptation service management function.

5) The application adaptation service management function forwards this request to the adaptation service control function using the service ready request message for referencing of the appropriate service execution engine.

6) The adaptation service control function forwards this request to the service environment adaptation function for the selection of the appropriate service execution engine.

7) The service environment adaptation function selects the requested service execution engine from the device profile.

8) The service environment adaptation function downloads the requested service execution engine to the user device.

9) After downloading, the service environment adaptation function sends the service execution engine download response message to the adaptation service control function.

10) The adaptation service control function forwards this response to the application adaptation service management function using the service ready response message.

11) The application adaptation service management function sends the service select response message to the user device.

\section{EXPERIMENTAL RESULT}

\subsection{Service scenario}

One of the use cases is that PSCF provides continuous service with application service profiles when a user moves from the home to the car and/or office. Suppose that the service registration and user registration are done previously. 


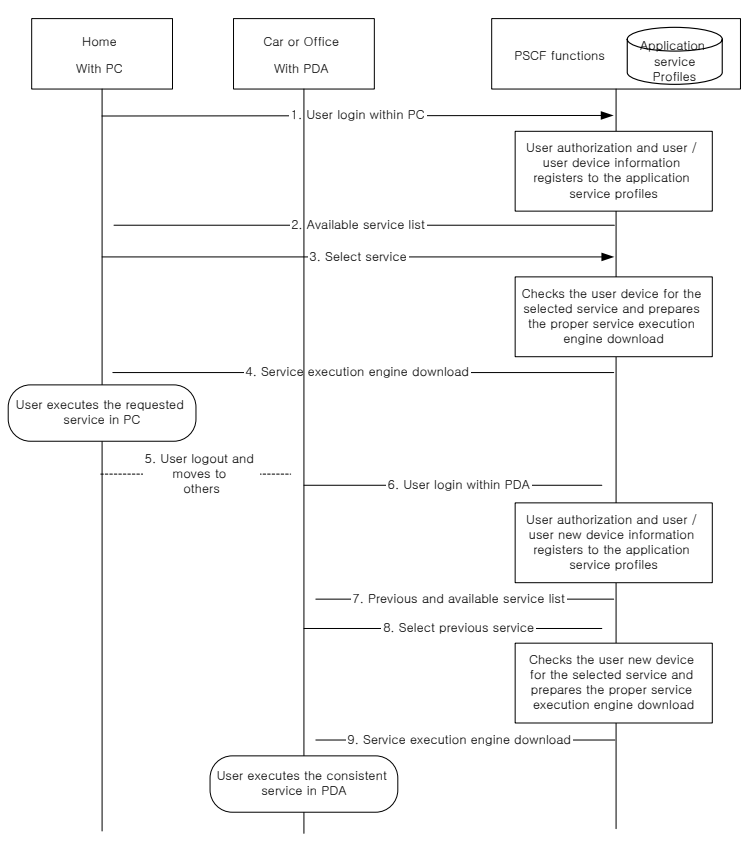

Fig. 8. Service scenario

Figure 8 describes the service scenario of service continuity when a user moves from the home with PC to the car or office with PDA. The flow of the scenario is as follows:

1) When a user requests login from PC, the PSCF function processes the user authorization. The user information and the user device information are registered to the session profile and the device profile in the application service profiles.

2) The PSCF function sends the available service list to the user device.

3) The user selects a service among available services from the list. The PSCF function checks if the user device has a candidate execution engine for the selected service using the service profile and the device profile. If not, a candidate service execution engine is selected for downloading.

4) The PSCF function downloads the service execution engine to the user device. The user executes the requested service in the PC.

5) The user logs out and moves to others and uses the different device (PDA).

6) When a user requests login from PDA, the PSCF function processes the user authorization for the new device. The user information and the new user device information are registered to the session profile and the device profile in the application service profiles.

7) The PSCF function sends to the new user device the previous service and new available service list.

8) The user selects the previous service. The PSCF function checks if the new user device has a candidate execution engine for the selected previous service using the service profile and the device profile. If not, a candidate service execution engine is selected for the download.

9) The PSCF function downloads the service execution engine to the new user device. The user executes the previous service continuously in PDA.

\subsection{Implementation environment}

We designed and implemented the proposed continuous video streaming service platform as shown in Figure $9 \sim 12$. Table 2 shows the implementation environments of client side and server side.

Table 2. The implementation environments

\begin{tabular}{|c|c|}
\hline - Client side & $\begin{array}{l}\text { - openSUSE11 / WinCE5.0 / } \\
\text { WinMobile6 classic } \\
\text { - GCC 3.2.1 } \\
\text { - eMbedded Visual C++4.0 } \\
\text { - FFplay/TCPMP 0.72RC1 media } \\
\text { player }\end{array}$ \\
\hline - Server side & $\begin{array}{l}\text { - Linux } 2.6 .9 \\
\text { - GNU C++ } \\
\text { - MySQL } 14.12 \\
\text { - Gtk+ } 2.0 \\
\text { - Apache HTTP Server. }\end{array}$ \\
\hline
\end{tabular}

Figure 9 demonstrates the adaptation of our system to the wired/wireless network on desktop Linux PC, WinCE PMP and WinMobile PDA.

A user is provided with HD (1024x768) level MPEG-4 VoD streaming content with 30 fps through PSCF server of Figure 10 when a user logs in on desktop PC in Figure 11. And then, a user stops or pauses the watching streaming content. After that, a user moves into hotspot and gets streaming continuity service with VGA (640x480) level MPEG-4, which is transformed to adapt to mobile device environment, with 15 fps on PMP from stop or pause scene as depicted in Figure 12. Also, the user has continuity service with QVGA (320x240) level MPEG-4 with 24 fps on PDA as depicted in Figure 12. Content scene in PC is prior to that of PMP and PDA. That is to say, this means that the user moved from PC to PMP or PDA. Here, the content scenes of PMP and PDA are identical. This shows video streaming service continuity.

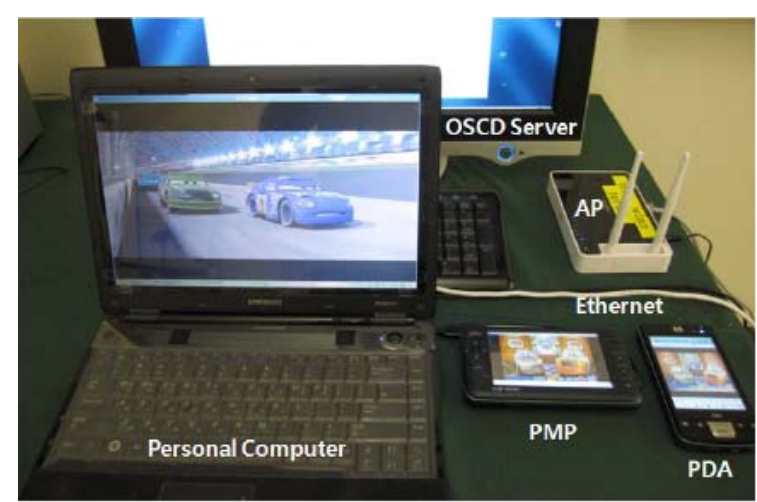

Fig. 9. Platform of video streaming continuous service 


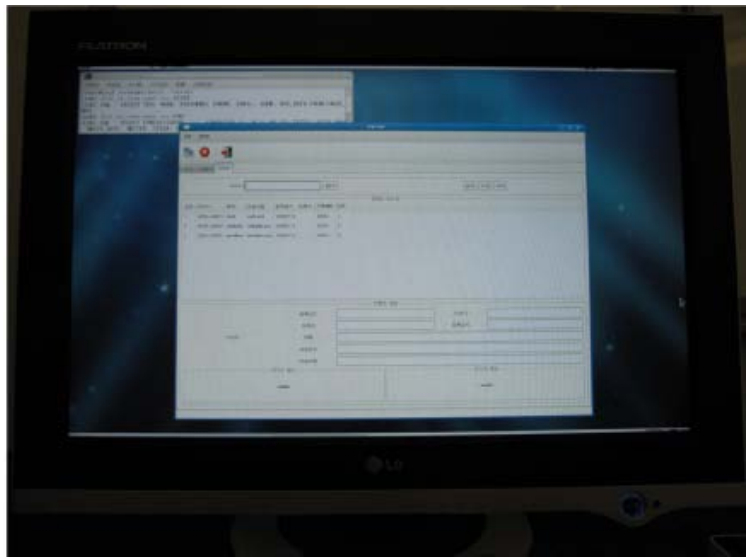

Fig. 10. Fedora based PSCF server

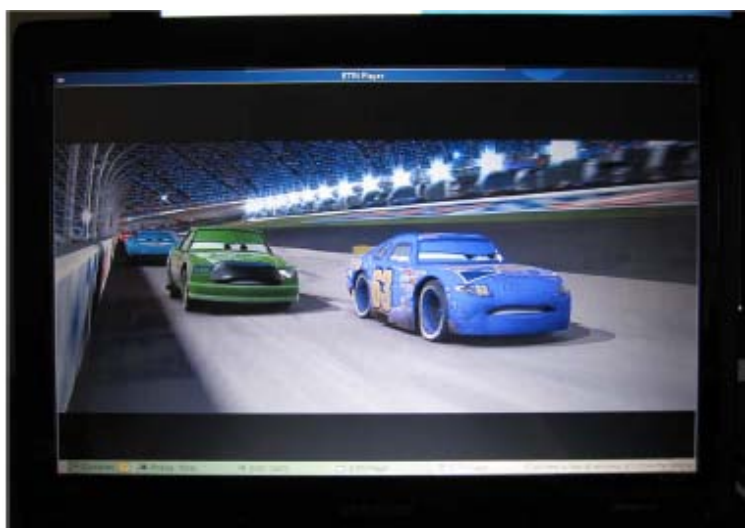

Fig. 11. openSUSE based PC client

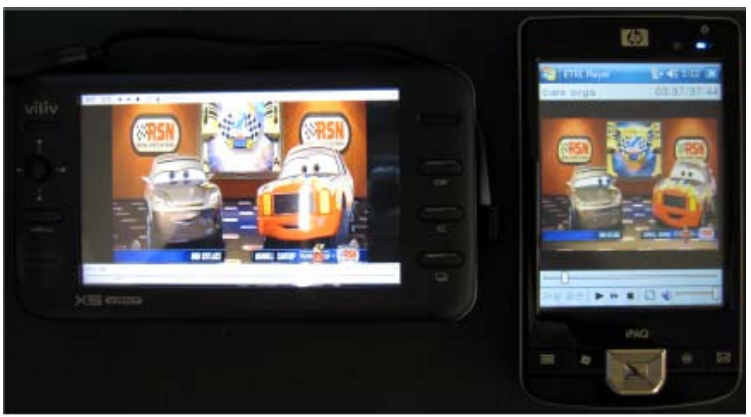

Fig. 12. WinCE based PMP client and WinMobile based PDA client

\section{CONCLUSION}

The dynamic adaptation between various service environments using the application profiles for the service continuity is a key issue of the profile-based service continuity framework for $\mathrm{N}$-screen service using next generation networks. PSCF offers an optimized service framework for providing continuous user services, which are multimedia video streaming, educational broadcasting, game, etc., using the various devices that are not restricted by the service environment of the user.

In this paper we focused on service continuity aspect for heterogeneous devices and proposed the profile-based service continuity framework offering the user seamless and convenient service continuity to improve his experience by enabling a more natural usage of his service. We presented a functional model and capabilities and also presented functional procedures for a cross different service environment based service continuity scenario. And finally, experimental results are presented. In this implementation, we prove the feasibility of our framework and the impact on the service and the user's experience.

\section{REFERENCE}

[1] Y. Huang, Tom Z. J. Fu, D. M. Chiu, John C. S. Lui, and Cheng Huang.,"Challenges, Design and Analysis of a Large-scale P2P-VoD System,” ACM SIGCOMM 2008, pp 375-388, 2008.

[2] Z. Chen, C. Lin, and X. Wei, "Enabling on-demand Internet Video Streaming Services to Multi-terminal Users in large scale," IEEE Transactions on Consumer Electronics, vol.55, no.4, pp.1988-1996, Nov. 2009.

[3] Y.-F. Chen, D. Gibbson, R. Jana, and H. Sun, "Project GeoTV - A Three-Screen Ser-vice: Navigate on SmartPhone, Browse on PC, Watch on HDTV," 6th IEEE Consumer Communications and Networking Conference, pp.1-2, Jan. 2009.

[4] H. Si, Y. Wang, J. Yuan, and X. Shan, "A Framework and Prototype for Service Mobility,” 2009 World Congress on Computer Science and Information Engineering, pp.315319, Mar. 2009.

[5] A.C. Snoeren, H. Balakrishnan, "An end-to-end approach to host mobility,” ACM MOBICOM 2000, pp. 155-166, August 2000.

[6] R. Jan, T. Raleigh, D. Yang, and L.F. Chang, "Enhancing survivability of mobile Internet access using mobile IP with location registers," IEEE INFOCOMM '99, vol. 1, pp. 3-11, 2000.

[7] M. Atiquzzaman, S. Fu, and W. Ivancic, "TraSH-SN: a transport layer seamless handoff scheme for space networks," Fourth Earth Science Technology Conference (ESTC), Crowne Plaza Cabana Palo Alto CA, June 2004.

[8] A. Di Stefano, C. Santoro, "NetChaser: agent support for personal mobility,” IEEE Internet Computing, pp. 74-79, 2000.

[9] M. Roussopoulos, P. Maniatis, E. Swierk, K. Lai, G. Appenzeller, and M. Baker, "Person-level routing in the mobile people architecture," USENIX Symposium on Internet Technologies and Systems, pp. 165-176, 1999.

[10] P. Maniatis, M. Roussopoulos, E. Swierk, K. Lai, G. Appenzeller, X. Zhao, and M. Baker, "The mobile people architecture," SIGMOBILE Mob. Comput. Commun. Rev., pp. 36-42, Jul. 1999.

[11] R. Liscano, R. Impey, Q. Yu, and S. Abu-Hakima, "Integrating multi-modal messages across heterogenous Networks," ENM-97, In Conjunction with the ICC-97, pp. 45-53, 1997.

[12] C. H. Herman Rao, D. F. Chang, Y. F. Robin Chen, and M. F. Chen, "iMobile: a proxy-based platform for mobile services," First Worshop on Wireless Mobile Internet, pp. 3-10, 2001.

[13] K. Raatikainen, "Middleware for future mobile networks," IEEE International Conference on $3 G$ Wireless and Beyond, pp.722-727, 2001.

[14] R. Bagrodia, S. Bhattacharyya, F. Cheng, S. Gerding, G. Glazer, R. Guy, Z. Ji, J. Lin,T. Phan, E. Skow, M. Varshney, and G. Zorpas, "iMASH: Interactive Mobile 
Application Session Handoff," ACM International Conference on Mobile Systems, Applications, and Services, May 2003.

[15] "Functional requirements and architecture of next generation networks," ITU-T SG13 Recommendation Y.2012, April 2010.

[16] "Resource and Admission control functions in next generation networks,” ITU-T SG13 Recommendation Y.2111, Nov. 2008.

[17] "Network attachment control functions in next generation networks,” ITU-T SG13 Recommendation Y.2014, March 2010.

[18] "Mobility management and control framework and architecture within the NGN transport stratum," ITU-T SG13 Recommendation Y.2018, Sep. 2009.

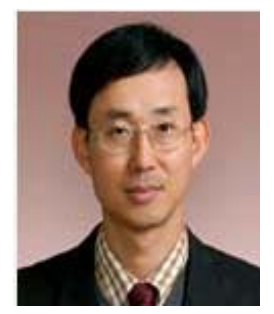

\section{Young-Sik Chung}

He received his B.S. degrees in computer science from Hongik University, Korea in 1983, and M.S. degrees in computer science from Hannam University, Korea in 1993. He joined the Electronics and Telecommunications Research Institute (ETRI) in 1983. He is currently working in the field of ubiquitous service framework technology, as a project leader of the Social Computing Research Team in ETRI. His current research interests include system software, communication software, multimedia service, and ubiquitous computing.

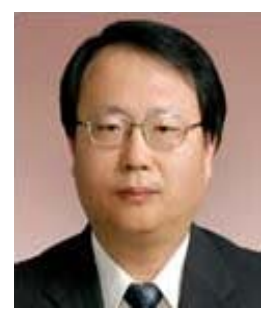

\section{Eui-Hyun Paik}

He received B.E, M.S, and Ph.D. degrees in Computer Science from SoongSil University, Seoul, Korea in 1984, 1987, and 1997, respectively. He joined the Electronics and Telecommunications Research Institute (ETRI) in 1987. He served as a principal member of the research staff in the network research center from that time until 2003. He is currently working in the field of social media service technology, as a team leader of the Social Computing Research Team in ETRI. His current research interests include social computing, IPTV, home networking, networking protocols, and intelligent agent technology.

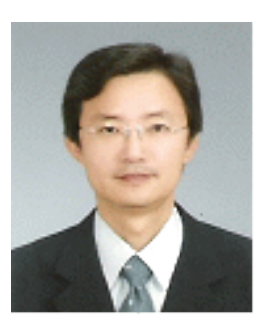

\section{Woo-Seop Rhee}

He received B.S. degree in computer science from Hong Ik University, Seoul, Korea, in 1983 and M.S., and Ph.D. degree in 1995 and 2003, respectively, in computer science from Chungnam National University, Daejeon, Korea.

From 1983 to 2005, he was with the Electronics and Telecommunication Research Institute (ETRI). He involved in development of TDX switching system, HANbit ACE ATM switching system and Optical access system as a project leader.

In 2005, He joined Hanbat National University, Daejeon, Korea and is currently associate professor of Multimedia engineering department. His research interests are concerned with broadband IPTV network architecture, quality of service in Internet and mobility management with multicast. $\mathrm{He}$ is an active member of ITU-T SG 13 as Editor and member of KICS, KOCON in Korea and IEEE. 